\title{
Expression and clinical significance of cytochrome c oxidase subunit IV in colorectal cancer patients
}

Kun Zhang ${ }^{1}$, Yibing Chen ${ }^{2}$, Xiaojun Huang ${ }^{2}$, Ping Qu ${ }^{2}$, Qiuzhong Pan ${ }^{3}$ Lin Lü3, Shanshan Jiang ${ }^{3}$, Tingitng Ren'2, Haichuan $\mathrm{Su}^{1}$

'Department of Oncology, Tangdu Hospital, Fourth Military Medical University, Xi'an, China

${ }^{2}$ State Key Laboratory of Cancer Biology and Experimental Teaching Center of Basic Medicine, Fourth Military Medical University, Xi'an, China

${ }^{3}$ Department of Biotherapy, Sun Yat-Sen University Cancer Center, Guangdong, China

Submitted: 16 December 2013

Accepted: 18 February 2014

Arch Med Sci 2016; 12, 1: 68-77

DOI: $10.5114 /$ aoms.2016.57581

Copyright @ 2016 Termedia \& Banach

\section{Abstract}

Introduction: Previous studies have demonstrated that the expression of cytochrome c oxidase (COX) subunits encoded by mitochondrial DNA is elevated in colorectal cancer (CRC). However, the expression of nuclear DNA-encoded COX IV and its clinical significance have not yet been investigated in CRC. Material and methods: We examined COX IV expression in paired CRC samples (cancer and pericancerous tissues) by quantitative real-time polymerase chain reaction (qPCR), Western blot and immunohistochemical staining and analyzed its clinical significance.

Results: QPCR and Western blot analyses showed that COX IV expression was significantly elevated at both the mRNA $(p=0.05)$ and protein levels in CRC tissue samples when compared with those in paired pericancerous tissues. Immunohistochemistry also revealed that COX IV expression was significantly increased in CRC tissues $(p<0.001)$. Association analyses showed that there was no significant association between COX IV expression and clinical parameters of CRC patients except for gender $(p=0.017)$. Moreover, we did not find any association between COX IV expression and overall survival or recurrence-free survival of CRC patients. Further analysis showed no significant relationship between the expression of COX IV and proliferating cell nuclear antigen (PCNA), a marker of cell proliferation.

Conclusions: Our findings suggest that elevated COX IV expression may play an important role in colorectal carcinogenesis, but not in progression, which warrants further investigation in future studies.

Key words: cytochrome c oxidase subunit IV, colorectal cancer, immunohistochemistry, real-time polymerase chain reaction.

\section{Introduction}

Colorectal cancer (CRC) is one of the most common malignancies worldwide and the third leading cause of cancer-related death in humans $[1,2]$. Nearly eight million new colorectal cancer cases are diagnosed each year worldwide. Despite great improvement in the treatment of CRC, the prognosis of patients remains poor. Current clinical and pathological parameters are not sufficient to precisely predict clinical outcome of CRC. Therefore, it is urgent to explore novel molecular biomarkers to discriminate patients with different molecular pathological profiles, thus

\author{
Corresponding author: \\ Tingitng Ren \\ State Key Laboratory \\ of Cancer Biology \\ and Experimental Teaching \\ Center of Basic Medicine \\ Fourth Military \\ Medical University \\ 169 West Changle St \\ 710032 Xi'an, China \\ Phone: +86-029-84774764 \\ E-mail: rtt419@fmmu.edu.cn
}


helping the elucidation of CRC carcinogenesis and the individualized treatment decision [3].

Mitochondria play a pivotal role in multiple physiological processes including energy production, catabolic and anabolic metabolism, generation of reactive oxygen species (ROS) and apoptosis. Mitochondrial dysfunction has long been implicated in the development of various malignancies, including CRC [4]. Somatic mitochondrial DNA (mtDNA) mutations, which are believed to be the consequence of ROS insult, have been observed in many types of cancers [5]. However, little is known about how mitochondrial alterations are involved in the process of carcinogenesis. Warburg first suggested that mitochondrial dysfunction-triggered aerobic glycolysis is the crucial metabolic pattern in cancer cells [6]. However, recent research has demonstrated that functional mitochondria are also important to support rapid growth of most tumor cells [7].

Previous analyses of mitochondrial dysfunction in cancer have typically focused on the alterations of mtDNA, including its content and mutations $[5,8,9]$. However, studies in the aging colon have demonstrated that immunostaining of cytochrome c oxidase (COX) is also a good surrogate indicator of mitochondrial function [10]. COX consists of 3 mtDNA-encoded and 10 genomic DNA-encoded subunits, forming complex IV of the electron transport chain [11]. In the aging colon, COX loss involves whole crypts, presumably as a consequence of mtDNA mutations that occur in the stem cells and then propagate along the crypt $[10,12]$. In addition, COX loss has been observed in colon epithelia insulted by ulcerative colitis, a premalignant lesion of colon cancer [13]. However, CRC cells show higher COX expression than either ulcerative or normal colon epithelia, indicating a complex role of COX expression alterations in the transformation of colon epithelia.

In the 13 subunits of COX, subunit IV (COX IV) plays a critical role in the assembly of COX. Although mtDNA exhibits a large copy number and high transcriptional activity [14], lower COX activity has been reported in a variety of mammalian cell types [15], implying the limiting role of COX IV in COX assembly. In previous studies, only mtDNA-encoded subunits I (COX I), II (COX II) and III (COX III) have been investigated in CRC [13]. However, expression level of the three subunits is not sufficient to reflect the activity of COX as an entire enzyme complex. Therefore, it is more useful to examine the expression of COX IV in exploring the biological functions of COX in CRC.

Nevertheless, to date, little is known about the biological functions of COX IV in CRC. We thus performed real-time PCR, Western blot and immunohistochemistry (IHC) to investigate the expres- sion of COX IV in CRC tissues [16]. In addition, we analyzed its association with clinicopathological parameters and prognostic value in CRC.

\section{Material and methods}

\section{Study population}

A total of 339 CRC patients who underwent $\mathrm{cu}$ rative surgery were consecutively recruited during the period from August 2008 to August 2012 in an ongoing epidemiological study at the Department of General Surgery, Xijing and Tangdu Hospitals, Fourth Military Medical University, Xi'an, China. Eligibility criteria of patient enrollment were as follows: a) colorectal adenocarcinoma diagnosed by histopathological examination; b) surgical treatment including colectomy and lymphadenectomy (limited or extended); c) availability of complete follow-up data; d) no preoperative treatment, such as chemotherapy and radiotherapy; e) no history of familial malignancy or other synchronous malignancy; and f) no death within 1 month after the operation. The histopathological type was determined using the criteria of the World Health Organization (WHO) classification. All the colorectal adenocarcinomas were graded based on their glandular differentiation degree, with grade 1 for well-differentiated adenocarcinomas (more than $95 \%$ of tumor composed of glands), including tubular adenocarcinomas; grade 2 for moderately differentiated adenocarcinomas (50-95\% of tumor composed of glands), and grade 3 for poorly differentiated carcinomas (49\% or less of tumor composed of glands). All patients were staged according to the seventh-edition Tumor-Node-Metastasis (TNM) staging system of the American Joint Committee on Cancer (AJCC). Postoperative follow-up, including physical and laboratory examinations, was performed at the outpatient department every 3 months for the first 2 years, every 6 months for the third to fifth years, and annually thereafter until at least 5 years after the operation or until the patient died, whichever came first. The last follow-up date was August, 2013. Informed consent was obtained from each patient.

This study was approved by the Ethical Committee of the Fourth Military Medical University and performed in accordance with the ethical standards of the Helsinki Declaration.

\section{Tissue samples}

For real-time quantitative polymerase chain reaction (PCR) analysis, a total of 23 paired cancerous tissues and matched adjacent pericancerous tissue located at least $2 \mathrm{~cm}$ away from the cancer were randomly selected. The 23 patients included 10 men and 13 women, with a median age of 73 years (range: $44-81$ years). 
For immunohistochemical staining, formalin-fixed, paraffin-embedded primary colorectal adenocarcinoma samples were collected from the 339 patients mentioned above and stored at room temperature. HE slides from these patients were viewed under a light microscope by pathologists and 3- $\mu \mathrm{m}$-thick tissue sections were cut from corresponding blocks containing representative tumor regions.

For Western blot analysis, there pairs of representative tissues were selected based on the IHC results (IHC-positive cancer tissues and IHC-negative pericancerous tissues).

\section{Quantitative real-time PCR}

Total RNA was extracted using TRIzol reagent (Invitrogen) according to the manufacturer's protocol. Then, two micrograms of RNA was reverse transcribed into first-strand cDNA by M-MLV Reverse Transcriptase (Promega) according to the manufacturer's instructions. COX IV and 18s rRNA were amplified by quantitative real-time $P C R$ using the following primers: COX IV forward: 5'-GAGTTGTATCGCATTAAGTTCAAGGAGAG-3', reverse: 5'-ACACATAGTGCTTCTGCCACATGATAA-3'; 18s rRNA forward: 5'-GACACGGACAGGATTGACAGATTGAT-3', reverse: 5'-ATGCCAGAGTCTCGTTCGTTATCG-3'. Gene-specific amplification was performed by the Bio-Rad CFX96 real-time PCR system. The relative expression of COX IV mRNA was normalized to the expression of 18s rRNA using $2^{-\Delta \Delta \mathrm{Ct}}$ method as previous described [17].

\section{Western blot}

Homogenized tissues were lysed in RIPA lysis buffer, and protein samples $(20 \mu \mathrm{g})$ were separated by electrophoresis in a $12 \%$ denaturing polyacrylamide gel. Blots were incubated with the primary antibodies at $4^{\circ} \mathrm{C}$ overnight, then incubated with secondary HRP-conjugated anti-rabbit antibody for $60 \mathrm{~min}$. The bands were detected with an enhanced chemiluminescence reagent (Millipore). Band density was measured with Quantity One image analysis software and was standardized to that of $\beta$-actin.

\section{Immunohistochemical staining}

Sections were incubated with antibody of COX IV (1 : 200, Abcam) or rabbit anti-human PCNA (1 : 2000, Cell Signaling Technology). The visualization signal was developed with the Invitrogen Histostain Plus kit. The intensity and extent of COX IV immunostaining were evaluated for all samples under double-blinded conditions. In brief, the percentage of positive staining was scored as 0 (0-9\%), 1 (10-25\%), 2 (26-50\%), 3 (51-75\%) or $4(76-100 \%)$, and the intensity as 0 (no stain- ing), 1 (weak staining), 2 (moderate staining) or 3 (dark staining). The total score was calculated as the product of intensity and extent, ranging from 0 to 12 .

\section{Statistical analysis}

All statistical analyses were performed using the SPSS Statistics 19.0 software (IBM). Differences in mRNA and protein expression between cancerous and pericancerous tissues were evaluated with the Wilcoxon test. The $\chi^{2}$ test was used to analyze the relationships between COX IV expression and various clinicopathological parameters. The Mann-Whitney $U$ test was used to compare the PCNA IHC score between patients with high and low COX IV expression. Kaplan-Meier survival function was calculated and compared with the log-rank test. The Cox proportional hazard regression model was used for univariate and multivariate analyses to explore the effects of the clinicopathological variables and COX IV expression on survival. All statistical tests were two-tailed and $p<0.05$ was considered to be significant.

\section{Results}

\section{COX IV expression was significantly} increased in CRC cells at mRNA and protein levels

We first measured COX IV mRNA levels in 23 paired cancerous and adjacent pericancerous tissues using quantitative real-time PCR. As shown in Figure $1 \mathrm{~A}$, COX IV mRNA expression level was higher in colorectal cancer tissues than that in the corresponding pericancerous tissues ( $p=0.05$ ), and $65.2 \%$ of the subjects (15/23) displayed higher COX IV mRNA expression in cancer tissues, compared with paired tissues.

To validate the qPCR findings, we performed immunohistochemical analyses of 339 colorectal cancer tissue blocks. As shown in Figure 2, COX IV expression was higher in cancer tissues than in adjacent noncancerous tissues ( $p<0.001$ ). We then determined COX IV protein levels in three pairs of representative samples by Western blot. Consistent with the quantitative real-time PCR and $\mathrm{IHC}$ results, Western blot analysis confirmed the significantly increased COX IV expression in CRC tissues when compared to their respective adjacent normal tissues (Figures 1 C, D).

\section{Up-regulated COX IV expression was located in cytoplasm and not associated with cell proliferation}

COX IV protein was mainly expressed in cytoplasm of both cancer cells and pericancer cells. Considering the elevated expression level of COXIV 
A

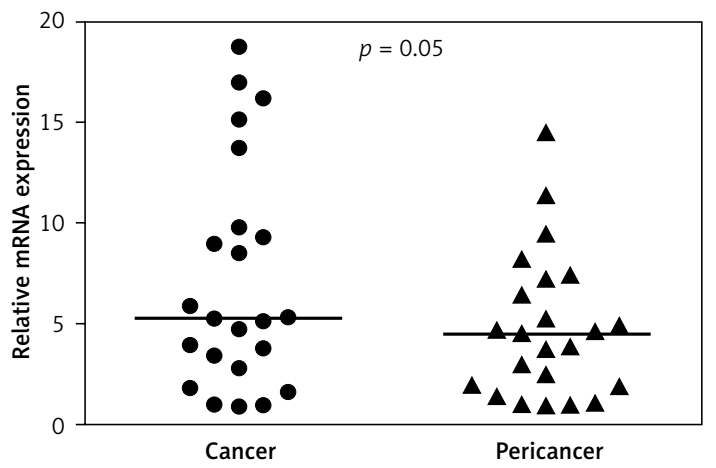

C

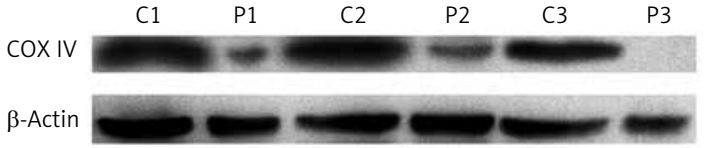

B

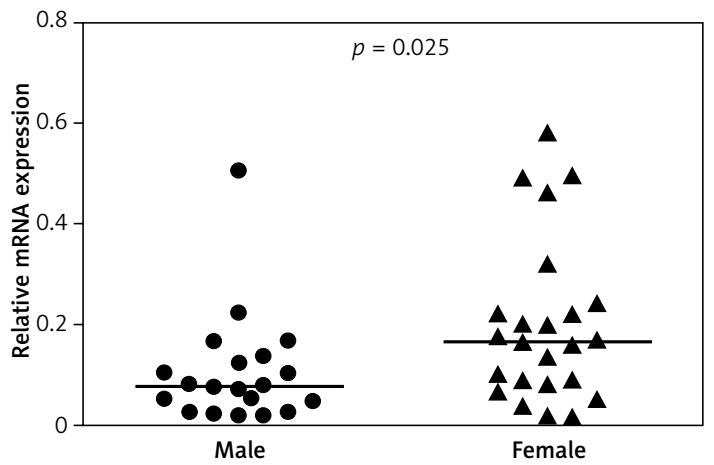

D

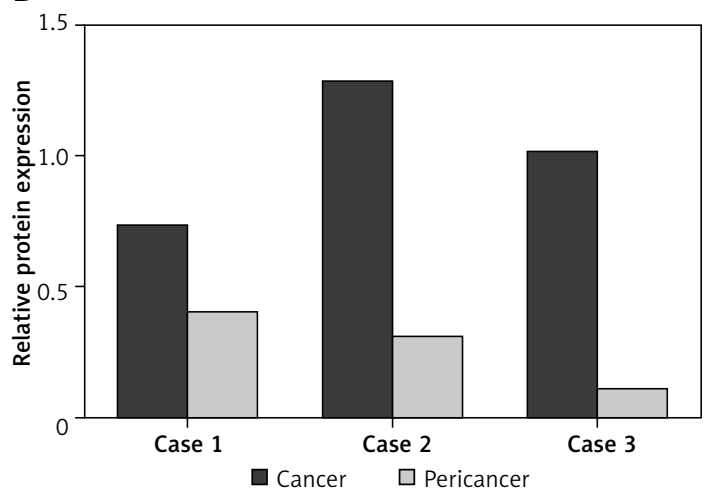

Figure 1. mRNA and protein expression of COX IV in CRC tissues. COX IV expression was significantly upregulated at both mRNA (A) and protein (C) levels in CRC tissues. Band intensity was quantified using Quantity One image analysis software (D). mRNA expression level of COX IV in male patients is lower than that in females (B)

in CRC, we hypothesized that elevated COX IV expression is involved in the carcinogenesis of CRC and thus explored the potential association between COX IV and PCNA expression. However, we failed to observe a significant association between expression of the two molecules (Figure $2 \mathrm{~K}$ ), implying the potential possibility that COX IV expression was not associated with CRC cell proliferation.

\section{COX IV expression was not associated with clinicopathological characteristics of CRC except gender}

There were 150 (44.2\%) female and 189 (55.8\%) male patients, with a median age of 63 years (range: 25-90 years). The median follow-up for the entire cohort was 57 months (range: 3-81 months). Among the 339 patients, 159 (46.9\%) were diagnosed with colon cancer and 215 (53.1\%) with stage I-II disease. After dividing patients into high or low expression subgroups with the median IHC score of COX IV in cancer tissues, we further analyzed the association between COX IV expression level and clinical characteristics of CRC patients. As shown in Table I, there was no significant association between COX IV expression and most patients' clinical characteristics. However, female patients showed a higher expression level than did males $(p=0.017)$, and this result is also confirmed by qPCR ( $p=0.025)$ (Figure $1 \mathrm{~B})$.

\section{COX IV expression was not associated with CRC patients' prognosis}

We also explored the prognostic value of COXIV expression in CRC. As shown in Figures $3 \mathrm{~A}, \mathrm{~B}$, there was no significant difference in either overall survival (OS) or relapse-free survival (RFS) between patients with low and high COX IV expression. Univariate Cox regression analyses revealed that OS was significantly decreased with higher TNM stage $(p<0.001)$, higher differentiation grade $(p=0.010)$, and high serum carcinoembryonic antigen (CEA) concentration $(p=0.036)$, while RFS significantly decreased with higher TNM stage $(p<0.001)$, higher differentiation grade $(p=$ $0.001)$, and CEA ( $p=0.003$ ) (Table II). Furthermore, multivariate Cox regression analyses confirmed TNM stage $(p<0.001)$ and differentiation grade $(p=0.009)$ as significant independent predictors of OS of CRC patients, whereas TNM stage ( $p<$ $0.001)$ and differentiation grade $(p=0.006)$ were significant independent prognostic factors for RFS (Table II). Considering different expression level of COX IV in male and female patients, we performed 


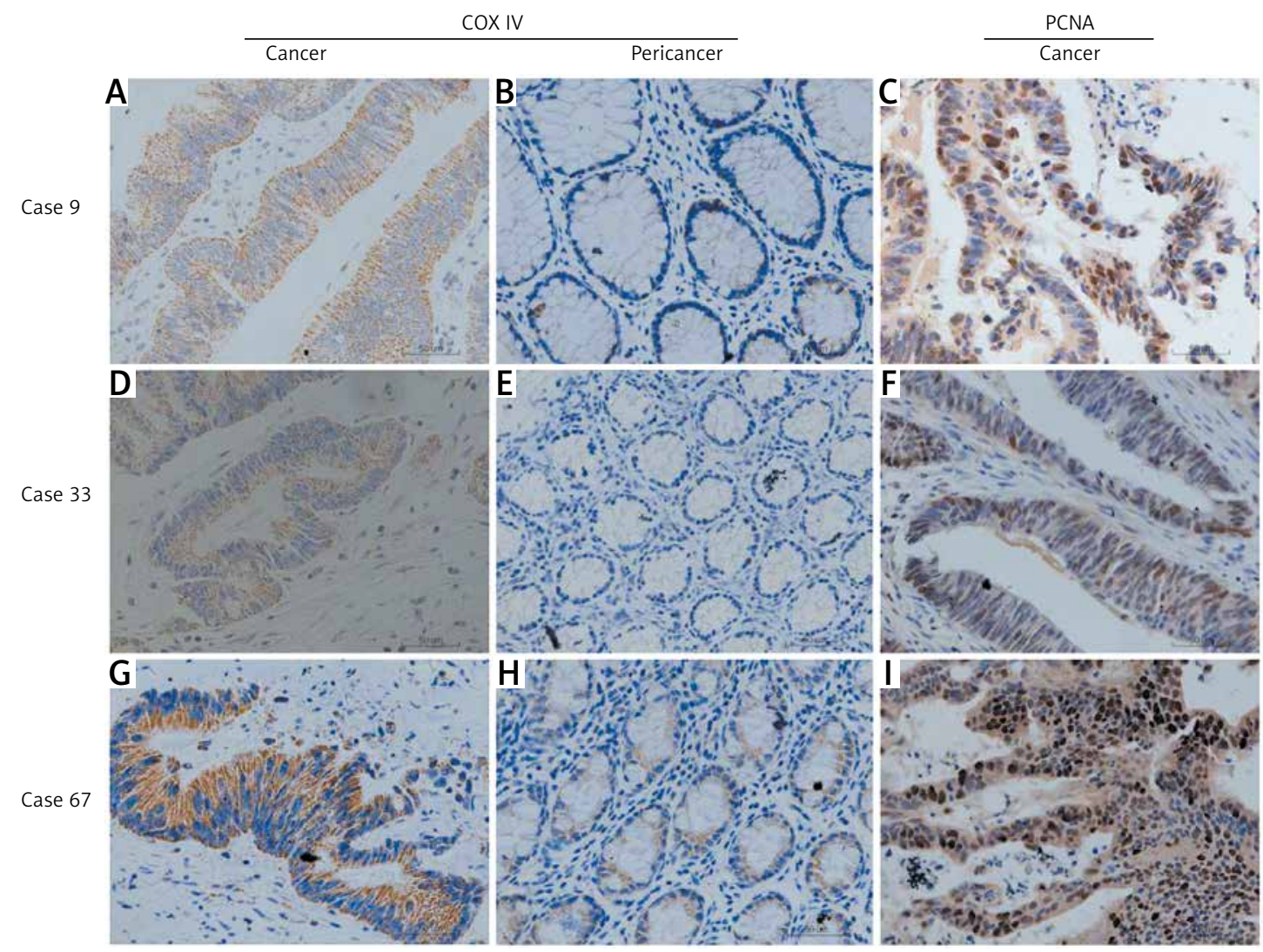

J

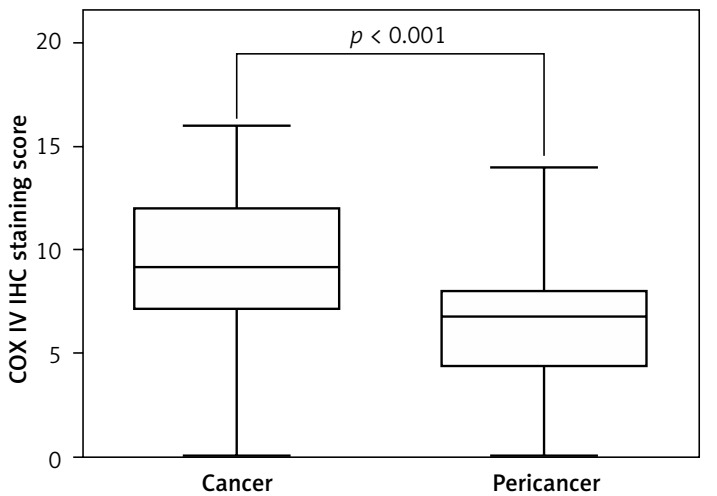

$\mathrm{K}$

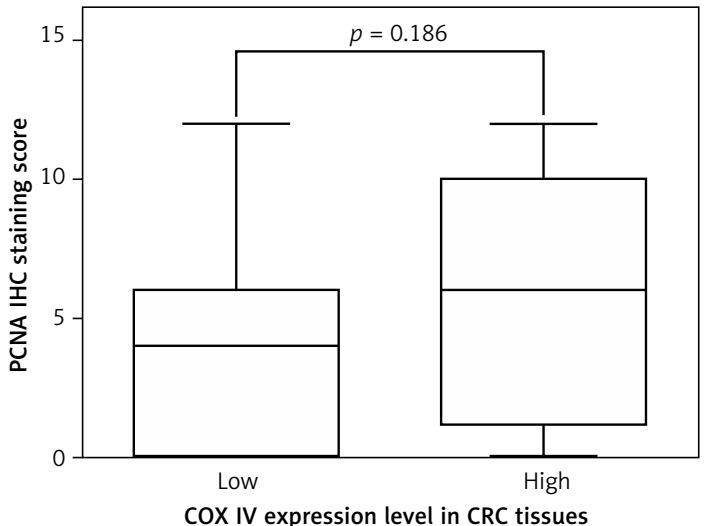

Figure 2. COX IV and PCNA expression in CRC tissues examined by immunohistochemistry. Representative intratumoral (A, D, G) and peritumoral (B, E, H) immunohistochemical staining of COX IV and representative intratumoral immunohistochemical staining of PCNA (C, F, I) are shown. Mann-Whitney test showed elevated COX IV expression in CRC tissues compared to pericancer tissues (J). In addition, there was no significant difference in PCNA expression between CRC samples with high or low COX IV expression (K)

survival analysis stratified by gender. As shown in Figures $3 \mathrm{C}-\mathrm{F}$, no prognostic significance of COX IV was observed in either gender.

\section{Discussion}

In this study, we examined the expression of COX IV and its association with clinical characteristics in CRC patients. We found that COX IV expression was elevated in CRC tissues when compared with adjacent noncancerous tissues. In addition, female patients showed higher COX IV expression than male patients. We also explored the prognostic value of COX IV in CRC patients. However, we observed no significant association between COX IV expression and clinical outcome of CRC patients.

COX is an important mitochondrial multi-protein complex with two main substrates - oxygen and cytochrome c [18]. The physiological role of COX as an electron carrier is well known; however, its biological functions in the development of can- 
Table I. Associations of COX IV expression with characteristics of CRC patients

\begin{tabular}{|c|c|c|c|c|}
\hline \multirow[t]{2}{*}{ Variables } & \multirow[t]{2}{*}{ No. of cases } & \multicolumn{2}{|c|}{ COX IV expression } & \multirow[t]{2}{*}{$P\left(\chi^{2}\right.$ test $)$} \\
\hline & & Low & High & \\
\hline Total & 339 & 167 & 172 & \\
\hline Age [years]: & & & & 0.780 \\
\hline$<63$ & 174 & 87 & 87 & \\
\hline$\geq 63$ & 165 & 80 & 85 & \\
\hline Gender: & & & & 0.017 \\
\hline Female & 150 & 63 & 87 & \\
\hline Male & 189 & 104 & 85 & \\
\hline Tumor locus: & & & & 0.424 \\
\hline Rectum & 180 & 85 & 95 & \\
\hline Colon & 159 & 82 & 77 & \\
\hline Tumor number: & & & & 0.976 \\
\hline Single & 335 & 165 & 170 & \\
\hline Multiple & 4 & 2 & 2 & \\
\hline Maximal diameter $[\mathrm{cm}]$ : & & & & 0.260 \\
\hline$<5$ & 160 & 84 & 76 & \\
\hline$\geq 5$ & 179 & 83 & 96 & \\
\hline CEA $[\mu \mathrm{g} / \mathrm{l}]:$ & & & & 0.477 \\
\hline$<10$ & 252 & 127 & 125 & \\
\hline$\geq 10$ & 87 & 40 & 47 & \\
\hline Grade: & & & & 0.464 \\
\hline I/II & 298 & 149 & 149 & \\
\hline III & 41 & 18 & 23 & \\
\hline TNM stage: & & & & 0.638 \\
\hline$|-| \mid$ & 215 & 108 & 107 & \\
\hline III-IV & 124 & 59 & 65 & \\
\hline Adjuvant therapy: & & & & 0.982 \\
\hline No & 57 & 28 & 29 & \\
\hline Yes & 282 & 139 & 143 & \\
\hline Death: & & & & 0.134 \\
\hline No & 252 & 104 & 148 & \\
\hline Yes & 87 & 28 & 59 & \\
\hline Relapse: & & & & 0.216 \\
\hline No & 236 & 97 & 139 & \\
\hline Yes & 103 & 35 & 68 & \\
\hline
\end{tabular}

CEA - carcinoembryonic antigen, COX IV - cytochrome coxidase subunit IV. 
A

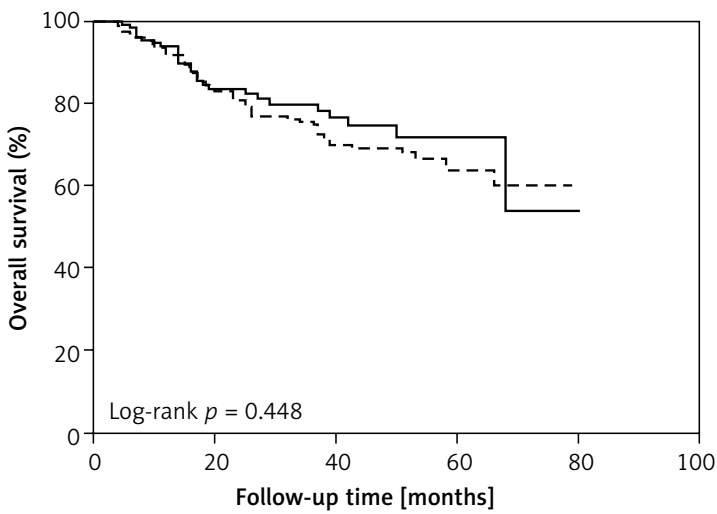

C

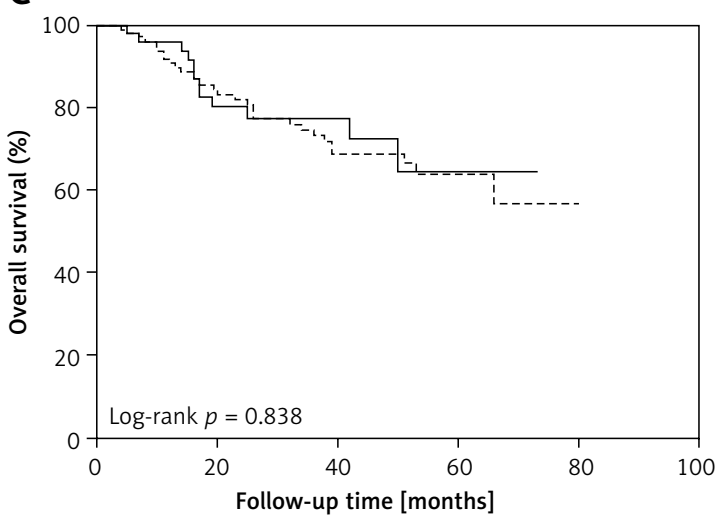

$\mathrm{E}$

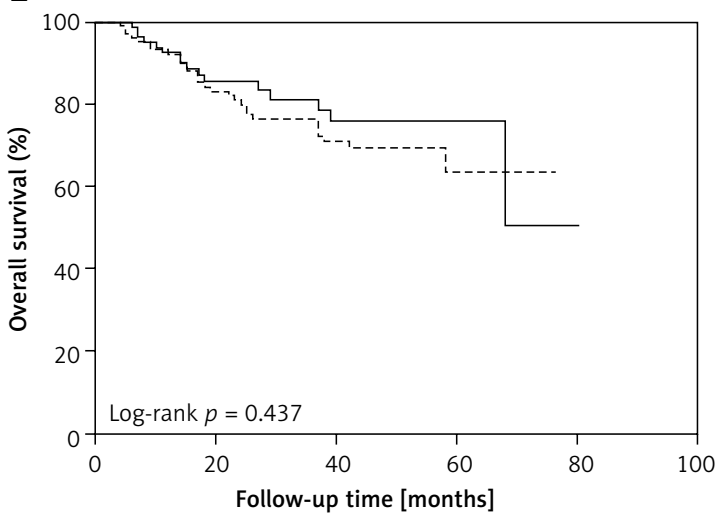

COX IV Low expression

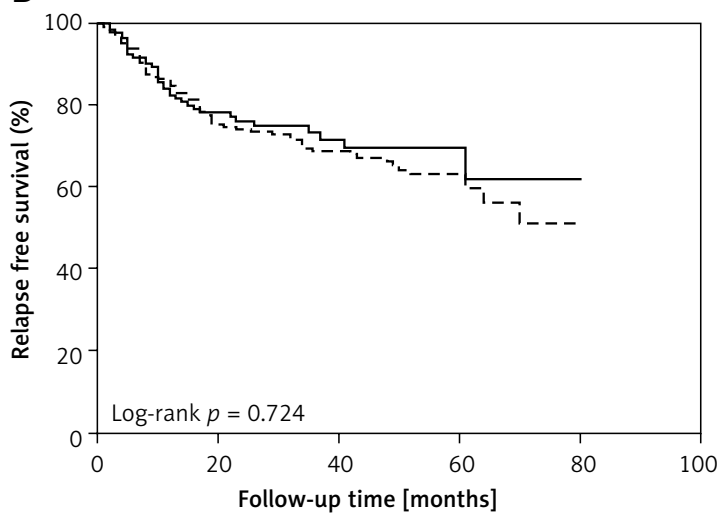

D

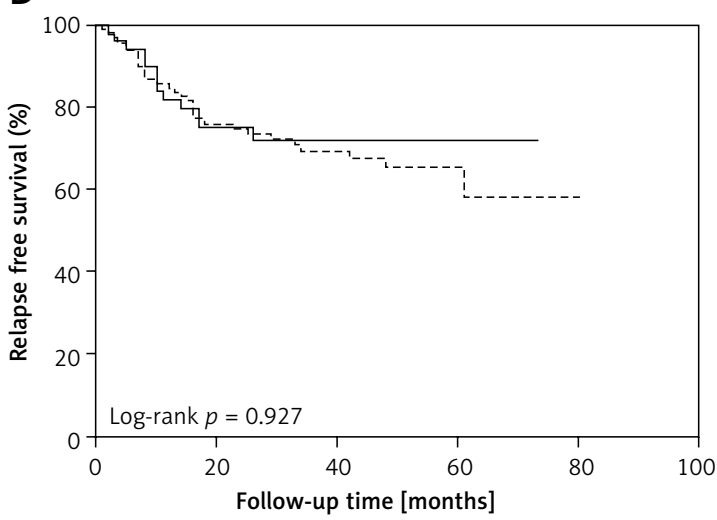

$\mathrm{F}$

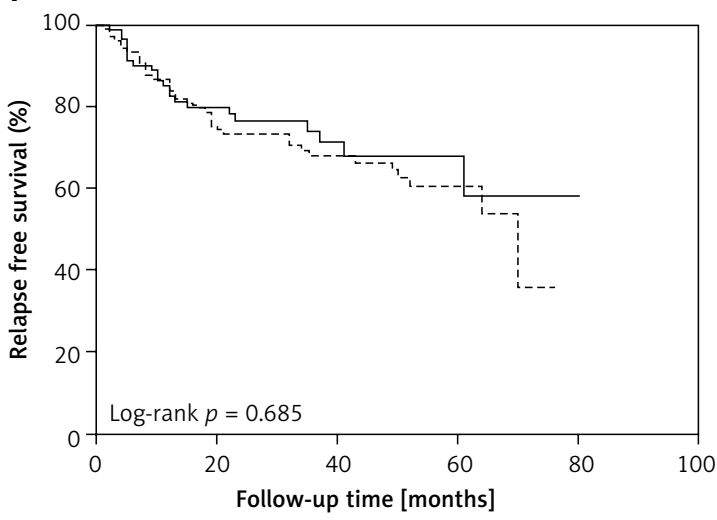

- - - COX IV High expression

Figure 3. Kaplan-Meier survival curves of patients with different COX IV expression levels. There was no significant difference in the OS (A) or RFS (B) of CRC patients with different COX IV levels. Furthermore, stratified analysis by gender showed that there was no significant difference in the OS (C for males and $\mathbf{E}$ for females) or RFS ( $\mathbf{D}$ for males and $\mathbf{F}$ for females) of CRC patients with different COX IV levels in either gender

cer have not been fully demonstrated. The alterations of COX in the pathogenesis of malignancies have been investigated in several studies. As the result of ROS injury accumulation, mutations of mtDNA-encoded subunits of COX (COXI to III) are well understood in a number of tumors, including CRC $[19,20]$, prostate cancer $[21,22]$, hepatocellular carcinoma [23] and myelodysplastic syndrome [24]. In addition to mtDNA-encoded COX subunits, genomic DNA-encoded subunits, especially COX V, are also studied in several tumor types. Increased expression of COX Va has been observed in lung cancer [25] and renal cell carcinoma [26]. Furthermore, the expression of COX I and III is repressed in premalignant colon epithelia but increased in CRC tissues [13]. In line with these findings, we observed the elevated COX IV expression in CRC tissues. These data collectively suggest that alterations of COX expression may play an important role in the pathogenesis of CRC. 
The cause of elevated COX IV expression in CRC remains unclear. Previous studies have demonstrated that the expression of COX II and IV is gradually depressed during the differentiation of murine erythroleukemia cells [27]. Therefore, it is not surprising that COX IV expression was upregulated in the development of CRC, a reverse procedure of differentiation. Indeed, Wu et al. demonstrated that autocrine gastrin increases $\mathrm{COX} \mathrm{Vb}$ expression in colon cancer HCT-116 cells [28]. In lung adenocarcinoma cells, it has been reported that induction of activated $\mathrm{H}$-Ras ${ }^{\mathrm{V} 12}$ enhances the expression of COX Vb [29]. In our study, we also found that female CRC patients showed higher COX IV expression than male patients. The higher prevalence of COX IV-overexpressed tumors in women, together with the lack of prognostic impact of COX IV expression in female CRC, indicates the possibility of a link between hormonal factors and COX IV expression status in CRC, and the underlying mechanisms of this impact need to be further elucidated. Therefore, an influence of anthropometric and lifestyle factors is also plausible and should be pursued in future studies.

Although there are several studies focused on the biological functions of COX units in the development of tumors, the role of elevated expression of COX IV in CRC remains to be elucidated. Chen et al. reported that knockdown of COX Va expression substantially suppresses the migration and invasion of non-small cell lung cancer (NSCLC) cells through inhibition of metalloproteinases MMP-2 and MMP-9 [25]. Moreover, elevated COX Va expression is associated with higher $\mathrm{N}$ stage and poorer prognosis of patients with lung adenocarcinoma. In glioblastoma multiform (GBM), increased COX Va activity has been associated with acquisition of temozolomide chemoresistance [30], as well as shorter progression-free survival and OS of patients [31]. Liang et al. also confirmed that COX Vb is involved in the metastatic potential of CRC cells [32]. Despite the important role of COX expression alteration in the carcinogenesis of colon epithelia [13], we observed no association of COX IV expression with proliferation and progression of CRC, as indicated by PCNA staining and TNM stage respectively. Furthermore, we failed to observe the prognostic value of COX IV expression. One explanation of these differences is that alterations of different COX subunits may contribute to different stages of CRC development. Proteomic studies have demonstrated that the ratio of nuclear encoded COX subunits to mtDNA-encoded COX subunits increases during the progression of carcinogenesis, suggesting the coordination of COX subunit alterations in the reprogramming of cancer metabolism [33, 34]. In addition, differences in study design, ethnic background, population sampling and experimental protocols may also account for the differences between our data and others.

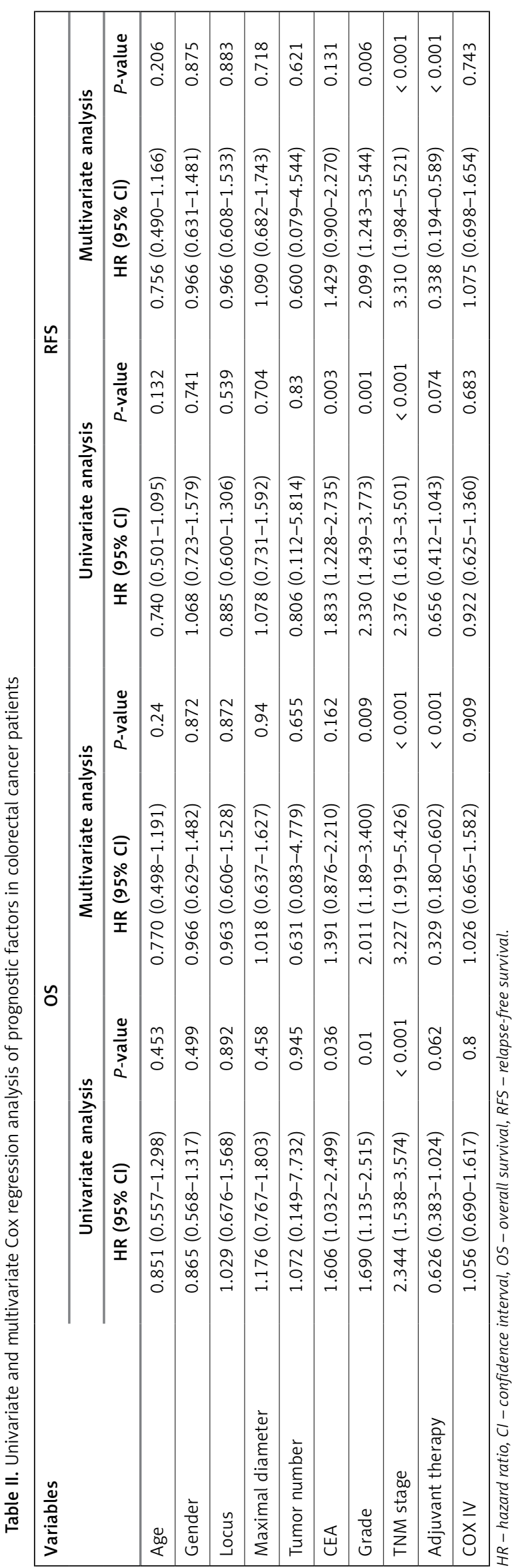


Therefore, the role of COX IV in the development of CRC needs to be further investigated in future studies.

In conclusion, our study for the first time demonstrated the elevation of COX IV expression levels in CRC tissues compared with adjacent nontumor tissues. In addition, the expression level of COX IV in female patients was higher than that in male patients. However, we did not find any association of COX IV expression with the progression of CRC or prognosis of patients. Our findings contribute to the current understanding of the COX subunit in the procedure of CRC development, which warrants further investigation in the alterations of the respiratory chain in CRC.

\section{Acknowledgments}

This work was supported by Program for New Century Excellent Talents in University, National Natural Science Foundation (81171966), and National Key Technologies R\&D Program (2011ZX09307-001-04) of China.

\section{Conflict of interest}

The authors declare no conflict of interests.

\section{References}

1. Jemal A, Bray F, Center MM, Ferlay J, Ward E, Forman D. Global cancer statistics. CA Cancer J Clin 2011; 61: 69-90.

2. Stec R, Plawski A, Synowiec A, Maczewski M, Szczylik C. Colorectal cancer in the course of familial adenomatous polyposis syndrome ("de novo" pathogenic mutation of APC gene): case report, review of the literature and genetic commentary. Arch Med Sci 2010; 6: 283-7.

3. Sanz-Pamplona R, Berenguer A, Cordero D, et al. Clinical value of prognosis gene expression signatures in colorectal cancer: a systematic review. PLoS One 2012; 7: e48877.

4. Wallace DC. Mitochondria and cancer. Nat Rev Cancer 2012; 12: 685-98.

5. Yu M. Somatic mitochondrial DNA mutations in human cancers. Adv Clin Chem 2012; 57: 99-138.

6. Warburg O. On the origin of cancer cells. Science 1956; 123: 309-14.

7. Ward PS, Thompson CB. Metabolic reprogramming: a cancer hallmark even warburg did not anticipate. Cancer Cell 2012; 21: 297-308.

8. Chatterjee A, Dasgupta S, Sidransky D. Mitochondrial subversion in cancer. Cancer Prev Res (Phila) 2011; 4: 638-54.

9. Houshmand M, Montazeri M, Kuchekian N, Noohi F, Nozar G, Zamani A. Is 8860 variation a rare polymorphism or associated as a secondary effect in HCM disease? Arch Med Sci 2011; 7: 242-6.

10. Taylor RW, Barron MJ, Borthwick GM, et al. Mitochondrial DNA mutations in human colonic crypt stem cells. J Clin Invest 2003; 112: 1351-60.

11. Michel H, Behr J, Harrenga A, Kannt A. Cytochrome c oxidase: structure and spectroscopy. Annu Rev Biophys Biomol Struct 1998; 27: 329-56.
12. Greaves LC, Preston SL, Tadrous PJ, et al. Mitochondrial DNA mutations are established in human colonic stem cells, and mutated clones expand by crypt fission. Proc Natl Acad Sci USA 2006; 103: 714-9.

13. Ussakli CH, Ebaee A, Binkley J, et al. Mitochondria and tumor progression in ulcerative colitis. J Natl Cancer Inst 2013; 105: 1239-48.

14. Attardi G, Schatz G. Biogenesis of mitochondria. Annu Rev Cell Biol 1988; 4: 289-333.

15. Villani G, Greco M, Papa S, Attardi G. Low reserve of cytochrome $\mathrm{c}$ oxidase capacity in vivo in the respiratory chain of a variety of human cell types. J Biol Chem 1998; 273: 31829-36.

16. Cheng J, Yu H, Wang L, Wang X, Shen G. Primary oral and maxillofacial liposarcoma: a clinicopathological and immunohistochemical study of eleven cases. Arch Med Sci 2012; 8: 316-23.

17. Qu Z, Jiang Y, Xu M, et al. Correlation of adrenomedullin with the erythropoietin receptor and microvessel density in hepatocellular carcinoma. Arch Med Sci 2015; 11: 978-81.

18. Ludwig B, Bender E, Arnold S, Huttemann M, Lee I, Kadenbach B. Cytochrome $\mathrm{C}$ oxidase and the regulation of oxidative phosphorylation. Chembiochem 2001; 2 : 392-403.

19. Namslauer I, Lee HJ, Gennis RB, Brzezinski P. A pathogenic mutation in cytochrome $c$ oxidase results in impaired proton pumping while retaining $\mathrm{O}(2)$-reduction activity. Biochim Biophys Acta 2010; 1797: 550-6.

20. Namslauer I, Brzezinski P. A mitochondrial DNA mutation linked to colon cancer results in proton leaks in cytochrome c oxidase. Proc Natl Acad Sci USA 2009; 106: 3402-7.

21. Namslauer I, Dietz MS, Brzezinski P. Functional effects of mutations in cytochrome $\mathrm{c}$ oxidase related to prostate cancer. Biochim Biophys Acta 2011; 1807: 1336-41.

22. Petros JA, Baumann AK, Ruiz-Pesini E, et al. mtDNA mutations increase tumorigenicity in prostate cancer. Proc Natl Acad Sci USA 2005; 102: 719-24.

23. Vivekanandan P, Daniel H, Yeh MM, Torbenson M. Mitochondrial mutations in hepatocellular carcinomas and fibrolamellar carcinomas. Mod Pathol 2010; 23: 790-8.

24. Reddy PL, Shetty VT, Dutt D, et al. Increased incidence of mitochondrial cytochrome c-oxidase gene mutations in patients with myelodysplastic syndromes. Br J Haematol 2002; 116: 564-75.

25. Chen WL, Kuo KT, Chou TY, et al. The role of cytochrome c oxidase subunit Va in non-small cell lung carcinoma cells: association with migration, invasion and prediction of distant metastasis. BMC Cancer 2012; 12: 273.

26. Yusenko MV, Ruppert T, Kovacs G. Analysis of differentially expressed mitochondrial proteins in chromophobe renal cell carcinomas and renal oncocytomas by 2-D gel electrophoresis. Int J Biol Sci 2010; 6: 213-24.

27. Vizirianakis IS, Pappas IS, Tsiftsoglou AS. Differentiation-dependent repression of c-myc, B22, COX II and COX IV genes in murine erythroleukemia (MEL) cells. Biochem Pharmacol 2002; 63: 1009-17.

28. Wu H, Rao GN, Dai B, Singh P. Autocrine gastrins in colon cancer cell up-regulate cytochrome $\mathrm{c}$ oxidase $\mathrm{Vb}$ and down-regulate efflux of cytochrome $c$ and activation of caspase-3. J Biol Chem 2000; 275: 32491-8.

29. Telang S, Nelson KK, Siow DL, et al. Cytochrome c oxidase is activated by the oncoprotein Ras and is required for A549 lung adenocarcinoma growth. Mol Cancer 2012; 11: 60 
30. Oliva CR, Nozell SE, Diers A, et al. Acquisition of temozolomide chemoresistance in gliomas leads to remodeling of mitochondrial electron transport chain. J Biol Chem 2010; 285: 39759-67.

31. Griguer CE, Cantor AB, Fathallah-Shaykh HM, et al. Prognostic relevance of cytochrome $C$ oxidase in primary glioblastoma multiforme. Plos One 2013; 8: e61035.

32. Liang L, Qu L, Ding Y. Protein and mRNA characterization in human colorectal carcinoma cell lines with different metastatic potentials. Cancer Invest 2007; 25: 427-34.

33. Krieg RC, Knuechel R, Schiffmann E, Liotta LA, Petricoin EF 3rd, Herrmann PC. Mitochondrial proteome: cancer-altered metabolism associated with cytochrome c oxidase subunit level variation. Proteomics 2004; 4: 2789-95.

34. Herrmann PC, Gillespie JW, Charboneau L, et al. Mitochondrial proteome: altered cytochrome c oxidase subunit levels in prostate cancer. Proteomics 2003; 3 : 1801-10. 